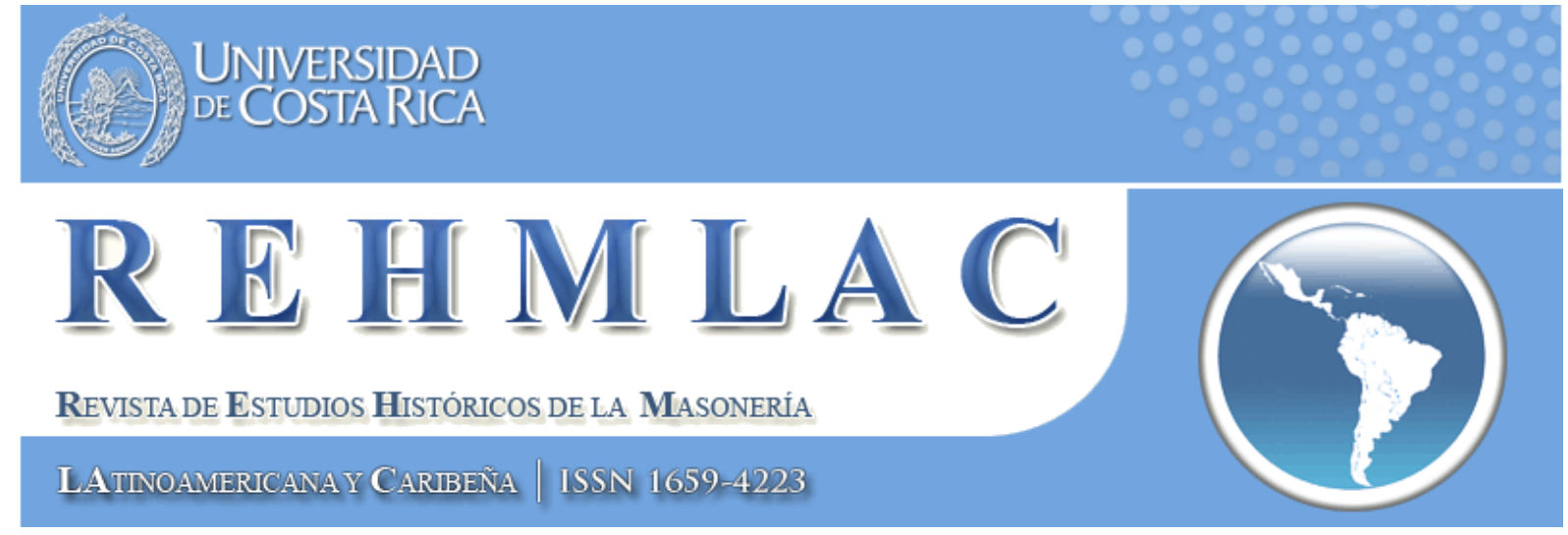

« La Franc-maçonnerie à l'épreuve de la Révolution française : Une expérience aux origines de la Francmaçonnerie libérale»

\author{
Éric Saunier
}

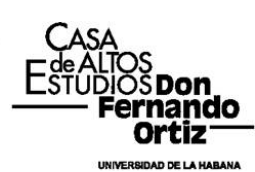


(C) Éric Saunier et REHMLAC.

\section{Équipe REHMLAC}

Conseil scientifique :

Miguel Guzmán-Stein (Universidad de Costa Rica, Costa Rica)

José Antonio Ferrer Benimeli (Universidad de Zaragoza, España)

Margaret Jacob (University of California Los Angeles, United States)

Eduardo Torres Cuevas (Universidad de La Habana, Cuba)

María Eugenia Vázquez Semadeni (Stanford University, United States)

Éric Saunier (Université du Havre, France)

Andreas Önnerfors (Lunds universitet, Sverige)

Samuel Sánchez Gálvez (Universidad Carlos Rafael Rodríguez de Cienfuegos, Cuba)

Roberto Valdés Valle (Universidad Centroamericana “José Simeón Cañas”, El Salvador)

Céline Sala (Université de Perpignan, France)

Dominique Soucy (Université de Franche-Comté, France)

Guillermo de los Reyes Heredia (University of Houston, United States)

Dévrig Mollès (Université de Strasbourg, France)

Felipe Santiago del Solar Guajardo (Universidad ARCIS, Santiago de Chile)

Carlos Francisco Martínez Moreno (Universidad Nacional Autónoma de México, México)

Michel Goulart da Silva (Universidade do Estado de Santa Catarina, Brasil)

Examinateurs de style:

Sylvia Hottinger (Centro de Estudios Históricos de la Masonería Española, España)

Kyle Jackson (University of California Los Angeles, United States)

Web administrateur et designer:

Luis Martín Valverde Alfaro (Universidad de Costa Rica, Costa Rica)

Editor:

Yván Pozuelo Andrés (IES Universidad Laboral de Gijón, España)

E-mail: yvan@edu.xunta.es

Director:

Ricardo Martínez Esquivel (Universidad de Costa Rica, Costa Rica)

E-mail: ricardo.martinezesquivel@ucr.ac.cr

Adresse web : www.rehmlac.com/

E-mail : info@rehmlac.com

PO Box : 243-2300 San José, Costa Rica 
Cité dans :

Academia.edu

Aladin. WRLC. Libraries Catalog

AFEHC. Asociación para el Fomento de los Estudios Históricos en Centroamerica

Biblioteca de Georgetown

CRICCAL, Université Sorbonne Nouvelle Paris 3

CERGE EI. Portál elektronických časopisů. Univerzita Karlova v Praze

Departamento de Filosofía de la Universidad Centroamericana "José Simeón Cañas"

Dialnet, Universidad de la Rioja

Directorio y recolector de recursos digitales del Ministerio de Cultura de España

DOAJ. Directory of Open Access Journals

e-revist@s. Plataforma Open Access de Revistas Científicas Electrónicas

Freemasonry and Civil Society Program at UCLA

Fudan University Library Academic Resource Portal

Google académico

Institute for the Study of the Americas at University of London

Latindex (UNAM)

Latindex.ucr. Repositorio de revistas de la Universidad de Costa Rica

Library Catalogue of University of South Australia

Museo Virtual de la Historia de la Masonería de La UNED

Nuevo Mundo. Mundos Nuevos

REDIAL. Red Europea de Información y Documentación sobre America Latina

SciELO - Scientific Electronic Library Online

Sherpa/Romeo

SID. Sistema Integrado de Documentación. Universidad Nacional de Cuyo

Toronto Public Library

UBO. Revues en ligne. Université de Bretagne Occidentale

Universia. Biblioteca de Recursos

University of Saskatchewan Library

University of Wiscosin-Madison Libraries

Western Theological Seminary. Beardslee Library Journals 


\title{
« La Franc-maçonnerie à l'épreuve de la Révolution française : Une expérience aux origines de la Franc-maçonnerie libérale »
}

\author{
Éric Saunier \\ Docteur en Histoire. Professeur UFR des Lettres et Sciences Humaines, université du Havre, France. \\ E-mail : eric.saunier@wanadoo.fr
}

Date de réception: 5 août 2014 - Date d'acceptation: 2 octobre 2014

Mots-clé :

Franc-maçonnerie, Révolution Française, Méthodologie, Historiographie, Premier Empire

\section{Keywords}

Freemasonry, French Revolution, Methodology, historiography, First Empire

\section{Résumé}

Après vingt ans de travaux réalisés en vue d'éclaircir la relation entre franc-maçonnerie et Révolution Française, il est temps d'extraire quelques enseignements concernant les expériences historiographiques. Ce qui nous permettra d'entrevoir les chemins qui s'ouvrent à nous dans la recherche sur cette sociabilité dans une des périodes les plus importantes de son histoire.

\begin{abstract}
After the work done in the last twenty years on the relationship between Freemasonry and the French Revolution, it is time to extract several lessons on historiography work and visualize the present and future paths of the history of this sociability in one of the key periods of its evolution.
\end{abstract}

\section{Introduction}

Cela fait plus de vingt ans que l'historiographie maçonnique de la période révolutionnaire semble avoir définitivement renoncé à prendre pour orientation de réflexion la question de l'influence des Francs-maçons et de la sociabilité maçonnique -orientation dont nous avons souvent souligné l'étonnante capacité à survivre ${ }^{1}$ - dans le déclenchement de la Révolution française. Depuis la thèse que nous avons soutenue au milieu des années 1990 sur les francs-maçons normands entre 1740 et $1830^{2}$ jusqu'à celle plus récemment présentée par Céline Sala sur les loges du Roussillon étudiées durant la même période ${ }^{3}$, le bilan qu'il est aujourd'hui possible de tirer de deux décennies de travaux sur la Franc-maçonnerie en Révolution fondés sur une approche méthodologique renouvelée ${ }^{4}$ a même abouti, parce que les chercheurs ont su exploiter la richesse des sources maçonniques et ont compris la plasticité

\footnotetext{
${ }^{1}$ Eric Saunier, « La Franc-maçonnerie est-elle à 1'origine de la Révolution française », dans : Les Francsmaçons (Paris : Fayard, 2011), 151-167; extrait de l'ouvrage : Les Francs-maçons de La Légende à l'histoire (Paris : Tallandier, 2003), 61-79.

${ }^{2}$ Saunier, Révolution et sociabilité en Normandie au tournant des XVIII ${ }^{e}$ et XIX ${ }^{e}$ siècles : 6000 francsmaçons de 1740 à 1830 (Presses universitaires de Rouen et du Havre (PURH), 1999).

${ }^{3}$ Céline Sala, Les francs-maçons en terres catalanes entre Lumières et Restauration. L'art royal de Perpignan à Barcelone (1740-1830) (Paris : H. Champion, 2009), 247-249.

${ }^{4}$ Eric Saunier, «La prosopographie: une nouvelle voie pour l'étude de la franc-maçonnerie », dans : Lumières, $n^{\circ}$, Franc-maçonnerie et politique au siècle des Lumières : Europe-Amériques, dir. Cecile Révauger (Presses universitaires de Bordeaux, 2006), 331-339. Saunier, "La prosopografía: una nueva vía para la historia de la masonería", dans : REHMLAC 1, no. 2 (diciembre 2009-abril 2010 [cité 17 Décembre, 2014]): disponible dans http://www.rehmlac.com/recursos/vols/v1/n2/rehmlac.vol1.n2-esaunier.pdf
} 
de ce lieu de sociabilité qui est devenu un observatoire de choix pour étudier les transformations sociétales occasionnées par la crise révolutionnaire, à un véritable renversement de la position originelle de recherche concernant ce binôme.

La plupart des travaux priorisent en effet aujourd'hui l'étude de ces transformations sociétales et ils le font avec d'autant plus d'intérêt que la Franc-maçonnerie a été l'un des rares endroits capables de rassembler à la veille de la Révolution les élites et la bourgeoisie moyenne dont on sait que l'étude des comportements et des représentations est un enjeu majeur de l'historiographie de la période révolutionnaire. De ces études engagées depuis près de vingt ans, il convient selon nous, dans le cadre de ce bilan synthétique, de présenter les trois enseignements principaux qui devraient fixer durablement l'orientation des travaux futurs. Le premier enseignement, apparu par le biais des apports des études critiques portant sur les versions successives des thèses conjuratrices, études qui ont eu pour préalable obligé de recourir à un élargissement chronologique allant du mitan des Lumières aux révolutions du premier XIX ${ }^{\mathrm{e}}$ siècle afin de saisir l'évolution de cette forme de sociabilité et le rôle qu'y a occupé véritablement la Révolution française, a été de mettre en lumière les très fortes résistances exprimées par cette forme de sociabilité devant l'arrivée de ces nouvelles pratiques culturelles qui, ayant pris naissance au temps des Lumières radicales ${ }^{5}$, se sont épanouies durant la décennie révolutionnaire. Le second a été (et c'est sans doute sur ce point que l'application à l'objet maçonnique de la méthode prosopographique a montré ses apports principaux), de faire émerger l'importance occupée par l'expérience révolutionnaire, cette expérience fut-elle éphémère et vécue par un petit nombre, dans la construction d'une identité maçonnique libérale qui allait être celle de toute la Franc-maçonnerie française jusqu'au début $\mathrm{du} \mathrm{XX}^{\mathrm{e}}$ siècle. Enfin, plus récemment, dans un contexte marqué par l'heureuse convergence du regain des études maçonniques sur la période napoléonienne ${ }^{6}$ et de l'intérêt croissant des historiens de la période napoléonienne ${ }^{7}$ envers la politique européenne élaborée sous le premier Empire, les travaux sur la franc-maçonnerie des années révolutionnaires permettent de rendre compte de l'importance occupée par la période du premier Empire dans l'internationalisation $\mathrm{du}$ processus de construction d'une Franc-maçonnerie libérale. Longtemps présentée comme le temps exclusif d'une instrumentalisation politique dont la croissance du nombre de francs-maçons et de loges serait le signe de la réussite ${ }^{8}$, cette période a en effet été celle de la mise en place d'un Grand Empire maçonnique ${ }^{9}$ dont il a résulté, en raison des mutations de sociabilité provoquées par la crise révolutionnaire dans les loges

\footnotetext{
${ }^{5}$ Jonathan I. Israel, Les Lumières radicales. La philosophie, Spinoza et la naissance de la modernité (16501750) (Editions Amsterdam, 2001).

${ }^{6}$ Voir notamment : Pierre Mollier dir., La Franc-maçonnerie : un âge d'or ? (Paris : Dervy, Collection « Renaissance traditionnelle $», 2007)$.

${ }^{7}$ Sur l'actualité de cette problématique, voir : François Antoine, Jean-Pierre Jessenne, Annie Jourdan et Hervé Leuwers dirs., L'Empire napoléonien : une expérience européenne? (Paris : A. Colin, " Recherches », 2013).

8 Cette vision est notamment diffusée dans la seule étude de synthèse sur la francmaçonnerie napoléonienne : François Collavéri, La Franc-maçonnerie des Bonaparte (Paris : Payot, rééd. 2007).

${ }^{9}$ Pierre-Yves Beaurepaire, L'Europe des francs-maçons, XVIII ${ }^{e}-X X^{e}$ siècles (Paris : Belin, 2002).
} 
françaises et de la diversité de réactions qu'elle a suscitée en Europe ${ }^{10}$, une nouvelle avancée des positions de cette franc-maçonnerie marquée par l'acceptation du politique et par une complète neutralisation religieuse.

\section{Les enseignements d'une réforme vue d'en bas : les limites de la modernité de la sociabilité maçonnique}

Le recul des thèses préconisant l'influence des Francs-maçons et des Loges dans le déclenchement de la Révolution, que ces thèses revêtent l'aspect du complot des arrière-loges proposé par Augustin de Barruel $^{11}$ ou celui de l'intégration de la sociabilité des loges dans le monde des sociétés de pensée qui détruisirent les fondements idéologiques de la Royauté défendue par Augustin Cochin ${ }^{12}$, constitue un aspect très remarquable de l'évolution de la recherche ${ }^{13}$. Toutefois, au-delà de la déconstruction nécessaire des thèses conjuratrices à laquelle se livraient déjà ses détracteurs éclairés dans les premières années ayant suivi la publication de l'ouvrage du jésuite ${ }^{14}$, les travaux récents sur la Franc-maçonnerie révolutionnaire ont pour intérêt principal d'avoir remis en cause un second aspect des représentations de la sociabilité maçonnique issues de la croyance dans l'origine francmaçonne du processus révolutionnaire. Il s'agit de l'idée selon laquelle la Franc-maçonnerie jugée naturellement d'esprit libéral en raison du contexte de sa naissance dans l'Angleterre sortie des guerres civiles et de l'adoption des Constitutions d'Anderson (1723) ${ }^{15}$ aurait été un

\footnotetext{
${ }^{10}$ Sur ce point, voir : Saunier, « La Franc-maçonnerie dans l'Europe napoléonienne. De l'échec de l'Europe maçonnique française à la transformation de la société des Maçons ", dans : REHMLAC 3, no. 1 (mayonoviembre 2011 [cité 17 Décembre, 2014]): disponible dans http://www.rehmlac.com/recursos/vols/v3/n1/rehmlac.vol3.n1-esaunier.pdf

${ }_{11}$ Augustin de Barruel, Mémoires pour servir à l'histoire du jacobinisme (Hambourg [Lyon], P. Fauché, $1797-$ $1798), 5$ vol.

${ }^{12}$ Voir : Augustin Cochin, «Les sociétés de pensée et la Révolution, II. La liberté », dans : Le Correspondant 94 (1922): 598-635. Cochin, La révolution et la Libre pensée. La socialisation de la pensée (1750-1789). La socialisation de la personne (1789-1792). La socialisation des biens (1792-1794) (Paris : Plon-Nourrit, 1924). Cochin, Les sociétés de pensée et la Révolution en Bretagne, 1788/1789 (Paris : H. Champion, 1925), 2 vol.

${ }^{13}$ Sur ce plan, l'étude récente de Daniel Kerjan sur l'histoire de la loge rennaise La Parfaite Union est d'un intérêt remarquable, l'atelier rennnais et le rédacteur du « Héraut de la nation » Michel-Ange Manugourit du Champ Daguet étant au centre de la dénonciation d'Augustin Cochin. Elle montre le caractère normatif de la participation desmaçons de La Parfaite Union aux événements révolutionnaires et l'ephémérité de la carrière maçonnique de Mangourit, celui-ci ayant quitté La Parfaite Union dix ans avant la Révolution. Daniel Kerjan met également en évidence la fragilité de la thèse cochinienne dans son étude des deux textes qui sous-tendent celle-ci. Le discours écrit le 23 juillet 1789 par Joseph Corbin de Pontbriand, procureur du Parlement et ex-vénérable de La Parfaite Union, quiserait la preuve d'un projet de complot en raison de l'évocation d'une union des maçons émerge comme un papier de circonstance à usage interne destiné à effacer les années de discorde entre les maçons rennais. Quant au texte de 1883 jugé par Cochin comme étant l'aveu de l'action conjuratrice des maçons rennais, il est l'œuvre d'un orateur séduit par le flatteur attribué à sa loge par les thèses barruéliennes.

${ }^{14}$ Jean-Joseph Mounier, De l'influence attribuée aux philosophes, aux francs-maçons et aux Illuminés sur la Révolution de France (Tübingen : J. G. Gotta, 1801), 1-18.

${ }^{15}$ Sur ce plan, l'influence des thèses du philosophe Jurgen Habermas sur le rôle exercé par la sociabilité francmaçonne dans la formation d'une société dominée par les valeurs culturelles de la bourgeoisie est particulièrement significative. Voir Habermas, L'espace public, archéologie de la publicité dans la dimension constitutive de la société bourgeoise (Paris : Payot, 1978).
} 
lieu de sociabilité servant de réceptacle naturel aux pratiques culturelles novatrices apparues avec le «tournant des Lumières» (Franco Venturi) des années 1770. Or, loin de conforter cette vision, la plupart des travaux engagés depuis vingt ans, lesquels accordent une place de choix à l'étude des pratiques des francs-maçons provinciaux qui avaient été proprement ignorée, montrent que, si les loges ont été le filtre par le biais duquel les Lumières descendirent socialement le plus bas, les réactions développées dans ces lieux de sociabilité qui s'étaient distingués par leur remarquable adaptation aux exigences culturelles imposées par une monarchie fondée sur l'absolutisme politique ${ }^{16}$ et sur l'exclusivisme religieux ${ }^{17}$, montrent qu'elles ont manifesté de fortes résistances devant la demande sociale exprimée en faveur de ces nouvelles pratiques culturelles fondées sur la reconnaissance de droits individuels et sur la démocratisation des cadres de vie associatifs.

C'est l'examen approfondi de la réponse donnée par les membres des loges de province et des ateliers coloniaux à la politique conduite par les dignitaires du Grand Orient à l'occasion de la réforme démocratique qui accompagne la naissance de la nouvelle obédience à la fin du règne de Louis XV (1771-1773) qui fournit l'illustration la plus claire d'une situation qui contredit la vision qui fut dominante jusqu'à la fin des années 1980, date à laquelle, après les travaux érudits de Pierre Chevallier sur la fondation du Grand Orient ${ }^{18}$, les analyses des relecteurs de Cochin sur le sens qu'il convenait de donner à la Réforme ${ }^{19}$, soulignaient encore, à partir d'une histoire vue d'en haut, les affinités entre les pratiques de la sociabilité maçonnique et les aspirations en faveur des libertés démocratiques revendiquées par la bourgeoisie qui dominait le recrutement des loges à la veille de la Révolution. Face à cette vision, les résultats de toutes les études engagées depuis vingt ans, qu'elles priorisent l'étude des discours des Francs-maçons en loge ou bien les pratiques destinées à répondre aux exigences imposées par la mise en place d'un appareil administratif construit pour répondre aux défis liés à l'engouement pour la sociabilité maçonnique, ont en effet mise en lumière les difficultés de celle-ci à s'adapter à cette nouvelle culture. Certes, ces travaux montrent que la Réforme survenue à la fin du règne de Louis XV a permis, comme l'ont montré ceux de Charles Porset sur la loge des Neuf Sœurs ${ }^{20}$ ou sur les Philalèthes de Paris ${ }^{21}$, l'émergence d'une nouvelle génération d'initiés qui étaient porteurs d'idéaux progressistes. Ils ont aussi montré que le choix de porter à la Grande Maîtrise de la nouvelle obédience le duc d'Orléans dont on sait maintenant qu'il s'intéressa de prêt à la vie maçonnique, sans justifier la thèse du complot orléaniste, témoigne de la montée en puissance d'un milieu libéral au sein de la

\footnotetext{
${ }^{16}$ Sur les rapports entre Franc-maçonnerie et pouvoir politique sous le ministère du cardinal de Fleury (17371747) puis de 1767 à 1771, voir : Pierre Chevalier, Le sceptre, la crosse et l'équerre sous Louis XV et Louis XVI (Paris : H. Champion, 1996).

${ }^{17}$ Le règlement général adopté par la première Grande Loge de France se concrétise ainsi par une restriction du libéralisme religieux andersonien (1735).

${ }^{18}$ Chevallier, Histoire de la franc-maçonnerie française 1 : La Maçonnerie, école de l'égalité (1725-1799) (Paris, Fayard, rééd. 1992).

${ }^{19}$ Voir notamment: François Furet, Penser la Révolution française (Paris : Gallimard, 1978). Ran Halévy, Les loges maçonniques aux origines de la sociabilité démocratique (Paris : A. Colin, 1984). Keith Baker, Au tribunal de l'opinion. Essais sur l'imaginaire politique au XVIII siècle (Paris : Payot, 1993), 9-44.

${ }^{20}$ Charles Porset, Une loge maçonnique d'avant 1789. La loge des Neuf Sœurs (Paris : Edimaf, 1989).

${ }^{21}$ Porset, Les Philalèthes et les convents de Paris, Une politique de la folie (Paris : H. Champion, 1996).
} 
franc-maçonnerie parisienne.

Toutefois, caractérisés par l'attention qu'ils portent à la manière dont les loges de province et des colonies ${ }^{22}$ se sont réappropriées la Réforme, ces travaux révèlent surtout l'importance des refus des Francs-maçons qui travaillèrent durant des dernières années de l'Ancien régime de répondre aux pratiques exigées par celle-ci et leur propension à défendre une conception de la sociabilité caractéristique de sociétés holistes ${ }^{23}$ préconisant des formes de solidarités collectives traditionnelles auxquelles le plus grand nombre des initiés à la Franc-maçonnerie manifestaient leur attachement.

Celui-ci se perçoit avec prégnance par le biais de l'étude des discours qui, comme l'a montré Céline Sala, témoigne d'une forte sensibilité des initiés à la Franc-maçonnerie au concept de Vertu morale, lequel est à la fois la raison principale de leur démarche initiatique ${ }^{24}$ et la valeur à laquelle ils sont le plus attachés. Sur le plan des pratiques, l'étude des scissions au sein des ateliers maçonniques, la mise en évidence de l'inclination des frères à maintenir des pratiques peu démocratiques, comme la perpétuation des vénérables dans leurs offices, plus encore celle d'une « culture de la distinction » qu'expriment à la fois le fort tropisme de l'Art royal sur la noblesse d'épée et l'élaboration de stratégies de réappropriation des hauts grades par la noblesse maçonnique à des fins de domination ${ }^{25}$, et le refus de la mixité sociale que reflète une étude affinée de l'organisation interne de la sociabilité maçonnique dans les grandes villes, sont autant de faits qui témoignent de la volonté d'un grand nombre d'initiés d'exprimer leur refus d'une forme de sociabilité nouvelle qui poserait les premiers jalons de la Franc-maçonnerie libérale.

Cette résistance devant la Réforme se manifeste aussi dans la propension croissante montrée par la société maçonnique à privilégier le recrutement d'hommes jouant un rôle de premier plan dans le maintien des cadres les plus traditionnels de la société d'Ancien régime. C'est en effet dans cette perspective que doit être regardée l'attraction (longtemps négligée) de la Franc-maçonnerie sur le monde de la prêtrise et sur la partie de la noblesse attachée à sa mission originelle. Fortement représentées dans les loges de province, les noblesses d'épée et de la rente foncière, dont l'influence contrebalance largement celle de la noblesse libérale dont l'action a été trop souvent mise en exergue en raison des fortes positions qu'elle occupe au sein de l'obédience parisienne, participent activement au maintien d'une sociabilité attachée aux hiérarchies et aux relations traditionnelles. Les affinités des Francs-maçons avec

\footnotetext{
${ }^{22}$ Saunier, "El espacio caribeño: un reto de poder para la francmasonería francesa", dans: REHMLAC 1, no. 1 $\begin{array}{llllll}\text { (mayo-noviembre } 2009 \quad \text { [cité } & 17 & \text { Décembre, } & 2014] \text { ): disponible dans }\end{array}$ http://www.rehmlac.com/recursos/vols/v1/n1/rehmlac.vol1.n1-esaunier.pdf

${ }^{23}$ Louis Dumont, Essais sur l'individualisme. Une perspective anthropologique sur l'idéologie moderne (Paris : Seuil, 1983).

${ }^{24}$ Sala, Les francs-maçons en terres catalanes.

${ }^{25}$ Ce phénomène émerge au grand jour à partir du milieu des années 1780 lorsque se met en place le Grand Chapitre Général. Voir : Saunier, "Les noblesses normandes et la Francmaçonnerie : diversité des cultures et culture de la distinction au XVIII ${ }^{e}$ siècle », dans : Les noblesses normandes (XVI $I^{e} X X^{e}$ s. $)$, dirs. A. Hugon et A. Boltanski (Presses universitaires de Rennes, 2011), 267-289. Pierre Mollier, La Chevalerie Maçonnique. Franc-maçonnerie, imaginaire chevaleresque et légende templière au siècle des Lumières (Paris : Dervy-Livres, 2005).
} 
les formes de sociabilité comme les confréries $^{26}$ et le monde des corporations ${ }^{27}$ en sont également de fortes expressions.

\section{De la Révolution française et de la naissance d'une Franc-maçonnerie libérale}

Avec l'émergence de limites importantes à la participation des loges à la modernisation des pratiques de sociabilité dans la France prérévolutionnaire, l'autre apport principal des travaux des historiens dans le domaine des études maçonniques est de donner à voir une nouvelle compréhension de la vie des Francs-maçons et des Loges durant les années 1789-1799, période qui ne fut pas seulement, à la différence de ce que pensaient les historiens qui avaient critiqué les thèses conjuratrices jusqu'au début des années 1980, uniquement un moment de destruction lié à l'inadaptation de cette forme de sociabilité à de nouvelles normes de comportement culture ${ }^{28}$ mais aussi un temps de dynamique de transformation née de l'appropriation de ces normes par les frères qui continuent de travailler dans leurs ateliers durant cette décennie ${ }^{29}$. Plus que les pratiques de subversion spectaculaires, tels la républicanisation des loges toulousaines ${ }^{30}$ ou le développement d'une Franc-maçonnerie parajacobine au Havre ${ }^{31}$, que la nécessaire critique de la thèse des origines franc-maçonnes de la Révolution a peut-être conduit à trop valoriser, les recherches récentes ont permis de souligner l'importance du "vécu de l'expérience révolutionnaire » dans la mise en place d'une Franc-maçonnerie qui, une fois transformée, allait construire le creuset de pratiques sur lequel allait se développer une Franc-maçonnerie d'essence libérale tout au long du XIX ${ }^{\mathrm{e}}$ siècle. Plus que l'action de nouveaux initiés éphémères qui ont trop souvent retenu l'attention en raison de leur notoriété, ce sont surtout des francs-maçons initiés avant la crise révolutionnaire qui, le plus souvent, comme ce fut le cas des membres des Amis de la Liberté $^{32}$, ont en effet imposé les règlements nouveaux dans les loges, choisi de refuser

\footnotetext{
${ }^{26}$ L'importance des appartenances communes entre les loges et les confréries de dévotion sont un apport important des études menées depuis vingt ans sur la sociabilité maçonnique de la fin du XVIII e siècle. Voir : Saunier, "Etre confrère et franc-maçon à la fin du XVIII ${ }^{\mathrm{e}}$ siècle », dans : Annales Historiques de la Révolution française 306 (1996): 617-635. Sala, "Sociétés de culture et culture de société : pénitents et francs-maçons en Catalogne sous l'Ancien Régime », dans : La Catalogne, une histoire méditerranéenne, un destin européen : dynamiques et modernités » (Paris, octubre 2008).

${ }^{27}$ Cette affinité émerge dans le contexte de la démocratisation de la sociabilité maçonnique survenue à partir du milieu des années 1780. Sur ce sujet, voir : Maurice Agulhon, Pénitents et Francs-maçons de l'ancienne Provence (Paris : Fayard, rééd. 1984). Saunier, Révolution et sociabilité, 67-76.

${ }^{28}$ Voir les développements d'Albert Soboul : Albert Soboul, "La Franc-maçonnerie et la Révolution », dans : Annales Historiques de la Révolution française (1974): 71-87.

${ }^{29}$ Voir notre mise au point : «Comment les Francs-maçons devinrent républicains ? », dans : $220^{e}$ anniversaire de la République (Paris : Ed. Conform, 2012), 29-37.

${ }^{30}$ Voir l'article « Loges républicaines », dans : Encyclopédie de la Franc-maçonnerie, Saunier dir. (Paris : L. G. F., rééd. 2000), 861-862.

${ }^{31}$ Voir Saunier, "Réaction et lieux de sociabilité en l'an III : l'exemple de la Franc-maçonnerie havraise », dans : Le Tournant de l'an III, Michel Vovelle dir. (Paris : CTHS, 1997), 411-425.

${ }^{32}$ Voir l'article : «Amis de la Liberté », dans : Encyclopédie de la Franc-maçonnerie, 25-26.
} 
d'enquêter sur les convictions religieuses d'un candidat à l'initiation ${ }^{33}$, ou, comme ce fut le cas au Havre, accepté d'installer une loge en pleine Terreur puis de la séparer en deux ateliers où le débat politique devint rapidement leur activité principale dans le contexte de la fermeture des sociétés populaires ${ }^{34}$. Deux ans avant cette évolution des loges havraises, non loin de là, à Caen, des francs-maçons initiés de longue date, majoritairement issus de la loge Union et Fraternité qui rassemblaient les élites protestantes, avaient il est vrai accepté de faire de cette loge l'embryon du Comité insurrectionnel qui devait diriger l'insurrection fédéraliste en Normandie ${ }^{35}$.

Ce processus de transformation de la sociabilité maçonnique, durable (les loges restèrent des cercles politiques sous le Premier Empire puis sous les monarchies censitaires) $)^{36}$, est loin de se limiter au seul domaine politique. Il a également touché l'approche de la spiritualité maçonnique qui constitue l'essence même de la démarche initiatique. C'est dans la France napoléonienne, où avec le Concordat de 1801 l'Eglise catholique a pourtant retrouvé de solides positions dans la société mais où la Franc-maçonnerie est cependant érigée " en culte bis $\gg{ }^{37}$, que l'on trouve les traces de cette transformation qui se caractérise par une neutralisation religieuse du Temple maçonnique. Dans cette évolution la crise révolutionnaire fut un tournant décisif car, à la fin de l'Ancien régime, dans un royaume de France où la franc-maçonnerie avait su d'adapter aux exigences imposées par le régime de l'Exclusivisme catholique, les relations étroites entre les loges et les prêtres, la difficulté de la philanthropie maçonnique à s'émanciper des gestes charitables comparables à ceux de l'Eglise, les précautions prises devant l'initiation des Protestants, témoignent d'une difficulté à accepter cette neutralisation religieuse pourtant préconisée implicitement par le texte d'Anderson. Sous la Révolution en revanche, le refus de s'immiscer dans le for intérieur religieux montré par l'attitude des vénérables de loges à l'occasion des initiations qui eurent lieu durant les vagues de déchristianisation de l'an $\mathrm{II}^{38}$ ou, à l'inverse, la propension montrée par d'autres vénérables à exposer, après la fin de cet épisode, les positions religieuses des impétrants pour leur signifier de les laisser à la porte du Temple ${ }^{39}$, sont les preuves de cette capacité de la crise

\footnotetext{
${ }^{33}$ Michel Taillefer, La franc-maçonnerie toulousaine, 1741-1799 (Paris : CTHS, 1984).

${ }^{34}$ Ces Loges sont celles que réinstalle le Grand Orient de France en 1774 et 1775 : La Fidélité et L'Aménité. Voir Saunier, « Réaction et lieux de sociabilité en l'an III », 411-425.

${ }^{35}$ Saunier, «Protestants et Francs-maçons au temps de la transition révolutionnaire (1750-1830): 1'exemple normand ", dans : Protestantisme et Franc-maçonnerie: de la tolérance religieuse à la religion de la tolérance?, Roger Dachez et Philippe Guglielmi (Paris : Editions maçonniques de France, 2000), 169-185.

${ }^{36}$ Sur le maintien de la culture politique libérale dans les loges de province sous le Premier empire et les monarchies censitaires, voir : Saunier, «La franc-maçonnerie et le libéralisme (1810-1827)», dans : Pour la révolution française, C. Le Bozec et E. Wauters dirs. (Presses Universitaires de Rouen - I.R.E.DC.H.R.C.T- Rouen, 1998), 399-407. Saunier, « La franc-maçonnerie entre cosmopolitisme et émergence de la conscience nationale ", dans : L'Europe à la recherche de son identité, Ch. Villain-Gandossi dir. (Paris : Editions du Comité des Travaux Historiques et Scientifiques, 2002), 247-258.

${ }^{37}$ Expression empruntée à Yves Hivert Messeca. Sur la laïcisation de la Franc-maçonnerie liée à l'instrumentalisation dont elle est l'objet à partir de 1802, voir, du même auteur : article " Laïcité ", dans : Encyclopédie de la Franc-maçonnerie, 475-478.

${ }^{38}$ Taillefer, La franc-maçonnerie toulousaine.

${ }^{39}$ Beaurepaire, «Le réveil des structures maçonniques locales sous le Directoire et le Consulat », dans : Du Directoire Consulat, 1. Le lien politique et social local sous le Directoire et e Consulat, Jacques Bernet, JeanPierre Jessenne et Hervé Leuwers dirs. (Lille : Septentrion, 1999), 97-110.
} 
révolutionnaire à faire avancer la laïcisation de la Franc-maçonnerie et à provoquer ce détachement du religieux qui lui permettra de devenir un acteur de premier plan dans le combat pour la consolidation de la République. En effet, ni le Premier empire, ni la monarchie restaurée, malgré des contextes idéologiques favorables, ne purent remettre en cause cette évolution ${ }^{40}$.

\section{Une expérience européenne}

Ladite évolution dépasse les frontières de la France pendant la période napoléonienne que l'on a trop souvent considérée, en raison de la croissance du nombre de loges, comme le temps d'un "âge d'or» où les Loges auraient été exclusivement l' "appareil idéologique d'état » dont rêvait Napoléon $\mathrm{I}^{\mathrm{er}}$ et qui aurait été en outre capables de maitriser la destinée d'ateliers que les loges françaises, vecteur de la construction de ce Grand Empire maçonnique $^{41}$, auraient su imposer aux états vassalisés ${ }^{42}$. Contre cette vision longtemps dominante, les études récentes mettent en effet en lumière une réalité maçonnique très différente. Cette différence se voit pour commencer, dans l'Empire français, où le développement d'une vie maçonnique qui, loin de répondre favorablement au projet $\mathrm{d}^{\prime}$ instrumentalisation ${ }^{43}$, parvient à maintenir son orientation libérale. Elle se voit aussi, dans les états vassalisés, dans la diversité des réactions devant la tentative d'exportation d'une Franc-maçonnerie d'Etat placée au service de l'Empire français. Au moment où la naissance de Grande Loge unie d'Angleterre (1813) entérine un modèle de vie maçonnique fondé sur l'exclusivité de pratiques initiatiques et d'actions charitables, cette diversité de réactions présente l'intérêt de révéler l'internationalisation des positions de cette franc-maçonnerie libérale qui avait été construite en France durant la décennie précédente. Cette avancée est d'autant plus remarquable que le projet napoléonien disposait de solides atouts pour faire triompher une sociabilité réduite aux fonctions festive et initiatique qui avaient été celles de la Franc-maçonnerie d'Ancien régime. En plus des aptitudes naturelles d'un lieu de sociabilité élitaire enraciné de longue date pour assimiler les élites européennes au régime impérial, en plus de la familiarisation de nombre de ces Francs-maçons à la Franc-maçonnerie d'Etat, il pouvait en effet s'appuyer sur les effets positifs de la préexistence de la politique menée par le Grand Orient de France depuis 1775. L'obédience voulait depuis cette date concurrencer les ambitions de la Franc-maçonnerie d'Angleterre qui, autour du concept de Maternité universelle, tentait alors d'imposer son hégémonie à nombre de Franc-maçonneries étrangères. De ce point de vue, la reconnaissance du Grand Orient de Pologne (1780) puis celle du Grand Orient de la République batave (1796) par le Grand Orient de France, doivent être considérées comme étant les préludes de la politique ayant donné naissance au Grand Orient de Bade (1804) et au Grand Orient de Westphalie (1808) dont les fondations durant les

\footnotetext{
${ }^{40}$ Voir l'article « Laïcité », dans : Encyclopédie de la Franc-maçonnerie, 475-478.

${ }^{41}$ Voir l'article « Grand Empire », dans : Encyclopédie de la Franc-maçonnerie, 349-250

${ }^{42}$ Collavéri, La Franc-maçonnerie des Bonaparte.

${ }^{43}$ Voir : Saunier, "La franc-maçonnerie en Normandie à l'époque de Napoléon », dans : Napoléon et la Normandie, Etudes Normandes 2 (2002) : 69-87.
} 
belles années impériales sont les exemples toujours mis en exergue pour illustrer la réussite de la politique napoléonienne. Dans ledit royaume de Westphalie attribué à Jérôme Bonaparte $^{44}$, il est particulièrement facile d'observer, à travers les hommages rendus à Bonaparte et le choix des titulatures, le succès de la construction de cette Franc-maçonnerie d'Etat, succès qui doit beaucoup à l'habilité du Grand Orient de France ${ }^{45}$, particulièrement attentif à respecter le « droit de territorialité » ${ }^{46}$.

Toutefois, aussi spectaculaires soient-ils, les exemples d'inféodation ne peuvent pas cacher l'existence d'une diversité de comportements qui, en raison de l'autonomie de positionnement dont elle témoigne, montre les avancées de la Franc-maçonnerie libérale, et cela y compris dans des pays qui, comme les états allemands, jouent le jeu de l'assimilation politique. Celle-ci ne peut pas en effet faire oublier le refus d'assimilation sociale manifesté par ces loges.

En Pologne, cette assimilation sociale est cependant une réalité que facilitent les liens historiques unissant depuis 1780 l'obédience française au Grand Orient de Pologne. Dans ce pays, c'est cependant l'assimilation politique qui fait défaut, comme le montrent l'évolution de la loge le Temple d'Isis et des loges fondées dans sa dépendance où l'on voit fleurir les emblèmes nationaux et réintroduire la pratique de la langue nationale, et cela malgré l'attitude loyaliste des premières loges de Varsovie. En Espagne où la Franc-maçonnerie compte pourtant à sa tête le ministre Azanza et où les Loges rassemblent un nombre important d'afrancesados, l'émergence d'un second Suprême Conseil du Rite Ecossais marqué par les influences anti-françaises en 1808 révéle de façon éclatante l'émergence d'un troisième modèle inhérent à la péninsule ibérique, un modèle caractérisé par un rejet total de la Francmaçonnerie napoléonienne, un rejet qui témoigne également des progrès d'une conception libérale de la Franc-maçonnerie.

\section{Conclusion}

Ainsi, des phénomènes de subversion de sociabilité apparus dès les premiers temps de la Révolution aux résistances qu'ont manifestées les Franc-maçonneries européennes face au projet de domination napoléonien, le regard que l'on peut porter aujourd'hui sur la

\footnotetext{
${ }^{44}$ Roi de Westphalie entre août 1807 et octobre 1813, Jérôme Bonaparte, initié dans une loge toulonnaise en 1801 (La Paix), confie dès son arrivée sur le trône à son ministre de l'Intérieur et de la Justice (et ancien Garde des Sceaux du GODF) Joseph Siméon le soin de diriger la maçonnerie westphalienne sur les bases d'une dépendance politique qui reproduit de manière identique celle qui fut imposée aux Maçons de France. C'est autour de la loge de Cassel rebaptisée Jérôme Napoléon à La Fidélité que se constitue le Grand Orient du Royaume de Westphalie.

${ }^{45}$ Les francs-maçons d'Anvers en créant une loge saint-Napoléon ne font que reproduire l'attitude des Frères de Vienne, lesquels constituèrent l'atelier saint-Joseph (Zur heiligen Joseph).

${ }^{46}$ Dans les anciennes Provinces-Unies, seules 9 des 80 loges sont dépendantes du Grand Orient de France, 71 loges d'entre elles reconnaissant l'autorité du Grand Orient de Hollande. Dans le prolongement des bonnes relations que l'obédience française entretenait avec le Grand Orient de Pologne qu'il contribua à fonder en 1780, le Grand Orient de France refuse également la constitution de la loge La Grande Armée en Pologne qui a émergé à Bromberg en 1811 avant la recréation du Grand Orient de Pologne en août 1812. Dans cette perspective, on peut citer l'exemple du rappel à l'ordre fait à la loge de Ljubjania qui choisit une titulature jugée trop ouvertement pro bonapartiste (Amis du Roi de Rome et de Napoléon).
} 
confrontation entre la Révolution et la sociabilité maçonnique, grâce aux apports de travaux ayant considérablement agrandi leurs cadres géographique et chronologique, se trouve radicalement transformé. L'idée de saisir les influences franc-maçonnes dans le déclenchement de la crise révolutionnaire cède la place à la réflexion sur les modes et les temps de construction des identités maçonniques. Une telle mutation doit-elle conduire les chercheurs à refermer définitivement le dossier de la Franc-maçonnerie en Révolution ? Nous ne le pensons pas. La complexité de l'interaction génère encore bien des zones d'ombre qu'il conviendra d'éclairer. Deux d'entre elles doivent être particulièrement soulignées. La première est la connaissance qui reste partielle du processus de transformation des pratiques maçonniques dans les loges françaises qui ont travaillé entre la chute des Tuileries et le coup d'état du 18 Brumaire. Les conclusions sont en effet fondées sur des études de cas ayant trait à la vie des seules loges parisiennes ${ }^{47}$ et de quelques espaces régionaux qui maintinrent une activité au paroxysme de la crise. Dans une perspective d'approfondissement, il conviendra d'étudier la vie des loges dans des villes comme Bordeaux et Lyon ${ }^{48}$, et de tirer le meilleur profit des richesses des archives revenues de Russie il y a plus de dix ans. Plus nécessaire encore est selon nous, du point de vue d'une histoire attentive aux constructions culturelles, la nécessité d'améliorer la connaissance de la réceptivité de la Franc-maçonnerie d'essence libérale dans le continent américain dont on connaît les liens avec l'histoire du développement des idéaux de liberté et de démocratie qui taraudent la Franc-maçonnerie française à partir des années révolutionnaires. Si l'on connaît les sympathies manifestées par les loges françaises devant la Révolution américaine et leur propension à accueillir ses principaux protagonistes, il faut maintenant explorer plus avant les pistes ouvertes par les quelques chercheurs qui se sont montrés attentifs à cerner la teneur des liens et l'impact des échanges culturels entre les Franc-maçonneries situées de part et d'autres des deux rives de l'Atlantique. C'est là la preuve s'il en est besoin que ce chantier mérite encore de nombreuses investigations.

\section{Bibliographie}

Agulhon, Maurice. Pénitents et Francs-maçons de l'ancienne Provence. Paris : Fayard, rééd. 1984.

Antoine, François, Jean-Pierre Jessenne, Annie Jourdan et Hervé Leuwers dirs. L'Empire napoléonien : une expérience européenne? Paris : A. Colin, « Recherches », 2013.

Baker, Keith. Au tribunal de l'opinion. Essais sur l'imaginaire politique au XVIIIe siècle. Paris : Payot, 1993.

Barruel, Augustin de. Mémoires pour servir à l'histoire du jacobinisme. Hambourg [Lyon], P. Fauché, 1797-1798.

Beaurepaire, Pierre-Yves. « Le réveil des structures maçonniques locales sous le Directoire et le Consulat ». Dans : Du Directoire Consulat, 1. Le lien politique et social local sous

\footnotetext{
${ }^{47}$ Voir l'article «Centre des Amis », dans: Encyclopédie de la Franc-maçonnerie, 129-130.

${ }^{48}$ André Combes, Histoire de la franc-maçonnerie à Lyon des origines à nos jours, Traboules (Lyon : Ed. Traboules, 2006).
} 
le Directoire et e Consulat. Dirigé par Jacques Bernet, Jean-Pierre Jessenne et Hervé Leuwers. Lille : Septentrion, 1999.

Beaurepaire, Pierre-Yves. L'Europe des francs-maçons, XVIIIe-XXe siècles. Paris : Belin, 2002.

Chevallier, Pierre. Histoire de la franc-maçonnerie française 1 : La Maçonnerie, école de l'égalité (1725-1799). Paris, Fayard, rééd. 1992.

Chevalier, Pierre. Le sceptre, la crosse et l'équerre sous Louis XV et Louis XVI. Paris : H. Champion, 1996.

Cochin, Augustin. "Les sociétés de pensée et la Révolution, II. La liberté ». Dans: Le Correspondant 94 (1922): 598-635.

Cochin, Augustin. La révolution et la Libre pensée. La socialisation de la pensée (17501789). La socialisation de la personne (1789-1792). La socialisation des biens (17921794). Paris : Plon-Nourrit, 1924.

Cochin, Augustin. Les sociétés de pensée et la Révolution en Bretagne, 1788/1789. Paris : H. Champion, 1925.

Collavéri, François. La Franc-maçonnerie des Bonaparte. Paris : Payot, rééd. 2007.

Combes, André. Histoire de la franc-maçonnerie à Lyon des origines à nos jours, Traboules. Lyon : Ed. Traboules, 2006.

Dumont, Louis. Essais sur l'individualisme. Une perspective anthropologique sur l'idéologie moderne. Paris : Seuil, 1983.

Furet, François. Penser la Révolution française. Paris : Gallimard, 1978.

Habermas, Jurgen. L'espace public, archéologie de la publicité dans la dimension constitutive de la société bourgeoise. Paris : Payot, 1978.

Halévy, Ran. Les loges maçonniques aux origines de la sociabilité démocratique. Paris : A. Colin, 1984.

Israel, Jonathan I. Les Lumières radicales. La philosophie, Spinoza et la naissance de la modernité (1650-1750). Editions Amsterdam, 2001.

Mollier, Pierre. La Chevalerie Maçonnique. Franc-maçonnerie, imaginaire chevaleresque et légende templière au siècle des Lumières. Paris : Dervy-Livres, 2005.

Mollier, Pierre dir. La Franc-maçonnerie : un âge d'or ? Paris : Dervy, Collection « Renaissance traditionnelle », 2007.

Mounier, Jean-Joseph. De l'influence attribuée aux philosophes, aux francs-maçons et aux Illuminés sur la Révolution de France. Tübingen : J. G. Gotta, 1801.

Porset, Charles. Une loge maçonnique d'avant 1789. La loge des Neuf Sœurs. Paris : Edimaf, 1989.

Porset, Charles. Les Philalèthes et les convents de Paris, Une politique de la folie. Paris : H. Champion, 1996.

Sala, Céline. "Sociétés de culture et culture de société : pénitents et francs-maçons en Catalogne sous l'Ancien Régime ». Dans : La Catalogne, une histoire méditerranéenne, un destin européen : dynamiques et modernités ». Paris, octubre 2008 . 
Sala, Céline. Les francs-maçons en terres catalanes entre Lumières et Restauration. L'art royal de Perpignan à Barcelone (1740-1830). Paris : H. Champion, 2009.

Saunier, Eric. "Etre confrère et franc-maçon à la fin du XVIIIe siècle ». Dans : Annales Historiques de la Révolution française 306 (1996): 617-635.

Saunier, Eric. « Réaction et lieux de sociabilité en l'an III : l'exemple de la Franc-maçonnerie havraise ». Dans : Le Tournant de l'an III. Dirigé par Michel Vovelle. Paris : CTHS, 1997.

Saunier, Eric. « La franc-maçonnerie et le libéralisme (1810-1827) ». Dans : Pour la révolution française. Dirigé par C. Le Bozec et E. Wauters. Presses Universitaires de Rouen - I.R.E.D-C.H.R.C.T- Rouen, 1998.

Saunier, Eric. Révolution et sociabilité en Normandie au tournant des XVIII e et XIX e siècles : 6000 francs-maçons de 1740 à 1830. Presses universitaires de Rouen et du Havre (PURH), 1999.

Saunier, Eric. « Protestants et Francs-maçons au temps de la transition révolutionnaire (17501830) : l'exemple normand ». Dans : Protestantisme et Franc-maçonnerie : de la tolérance religieuse à la religion de la tolérance? Dirigé par Roger Dachez et Philippe Guglielmi. Paris : Editions maçonniques de France, 2000.

Saunier, Eric dir. Encyclopédie de la Franc-maçonnerie. Paris : L. G. F., rééd. 2000.

Saunier, Eric. « La franc-maçonnerie entre cosmopolitisme et émergence de la conscience nationale ». Dans : L'Europe à la recherche de son identité. Dirigé par Ch. VillainGandossi. Paris : Editions du Comité des Travaux Historiques et Scientifiques, 2002.

Saunier, Eric. " La franc-maçonnerie en Normandie à l'époque de Napoléon ». Dans : Napoléon et la Normandie, Etudes Normandes 2 (2002) : 69-87.

Saunier, Eric. Les Francs-maçons de La Légende à l'histoire. Paris : Tallandier, 2003.

Saunier, Eric. « La prosopographie : une nouvelle voie pour l'étude de la francmaçonnerie ». Dans: Lumières, $n^{\circ}$ 7, Franc-maçonnerie et politique au siècle des Lumières : Europe-Amériques. Dirigé par Cécile Révauger. Presses universitaires de Bordeaux, 2006.

Saunier, Eric. "El espacio caribeño: un reto de poder para la francmasonería francesa". Dans: REHMLAC 1, no. 1 (mayo-noviembre 2009). Disponible dans http://www.rehmlac.com/recursos/vols/v1/n1/rehmlac.vol1.n1-esaunier.pdf

Saunier, Eric. "La prosopografía: una nueva vía para la historia de la masonería", dans : REHMLAC 1, no. 2 (diciembre 2009-abril 2010). Disponible dans http://www.rehmlac.com/recursos/vols/v1/n2/rehmlac.vol1.n2-esaunier.pdf

Saunier, Eric. « La Franc-maçonnerie dans l'Europe napoléonienne. De l'échec de l'Europe maçonnique française à la transformation de la société des Maçons ». Dans : REHMLAC 3, no. 1 (mayo-noviembre 2011). Disponible dans http://www.rehmlac.com/recursos/vols/v3/n1/rehmlac.vol3.n1-esaunier.pdf

Saunier, Eric. « Les noblesses normandes et la Francmaçonnerie : diversité des cultures et culture de la distinction au XVIIIe siècle ». Dans : Les noblesses normandes (XVIeXXe s.). Dirigé par A. Hugon et A. Boltanski. Presses universitaires de Rennes, 2011. 
Saunier, Eric. « Comment les Francs-maçons devinrent républicains ? ». Dans : 220e anniversaire de la République. Paris : Ed. Conform, 2012.

Soboul, Albert. « La Franc-maçonnerie et la Révolution », Dans : Annales Historiques de la Révolution française (1974): 71-87.

Taillefer, Michel. La franc-maçonnerie toulousaine, 1741-1799. Paris : CTHS, 1984. 